\section{Melanoma's hidden act}

\section{By Kai-Jye Lou, Staff Writer}

Researchers at the University of Bonn and the Johannes Gutenberg University Mainz have shown that melanoma cells can acquire resistance to adoptive $\mathrm{T}$ cell transfer therapies by dedifferentiating themselves to hide antigens. ${ }^{1}$ The group now is trying to circumvent this resistance mechanism and thinks doing so could improve treatment responses for current $\mathrm{T}$ cell therapy protocols.

Adoptive $\mathrm{T}$ cell transfer is one of three immunotherapy-based strategies used to treat metastatic melanomas and typically is used in patients for whom standard treatments have failed.

The two other immunotherapy approaches being developed for melanoma involve nonspecific immunostimulation and active immunization.

The two marketed immunotherapies for melanoma work via nonspecific immunostimulation: Proleukin aldesleukin IL-2 from Novartis AG and Yervoy ipilimumab, a human mAb against CTLA-4 (CD152) receptor from Bristol-Myers Squibb Co. Both drugs cause tumor regression in up to $15 \%$ of patients. ${ }^{2,3}$

The two most advanced active immunizers for melanoma are talimogene laherparepvec from Amgen Inc. and GSK1572932A from GlaxoSmithKline plc. Talimogene laherparepvec is a modified herpes simplex virus type 1 (HSV-1) encoding granulocyte macrophage colony-stimulating factor (GM-CSF; CSF2). GSK1572932A is a vaccine against melanoma-associated antigen A3 (MAGEA3). Both compounds are in Phase III testing.

Adoptive cell transfer typically involves isolating, expanding and activating tumor-infiltrating lymphocytes or peripheral blood $\mathrm{T}$ cells and then infusing the cells back into the patient.

Although peripheral blood T cells are more plentiful and are easier to isolate than tumor-infiltrating lymphocytes, they lack tumor specificity and thus need to be engineered to express $\mathrm{T}$ cell receptors (TCRs) or chimeric antigen receptors that target tumor antigens before they are infused back into the patient.

Companies have avoided adoptive cell therapies because they need to be tailored to each patient. Clinical trials evaluating adoptive cell therapies are being run by research institutes, including the National Cancer Institute (NCI).

NCI trials have shown objective tumor regression in up to $72 \%$ of patients. ${ }^{4,5}$ Response durations can range from just two months to over six years. The mechanisms underlying how melanomas develop resistance to these therapies over time are unclear.
A research group led by Thomas Tüting, a professor of experimental dermatology and head of dermato-oncology at University Hospital Bonn, has been trying to better understand how melanomas acquire resistance to adoptive $\mathrm{T}$ cell therapies. Understanding such mechanisms could help improve the durability of the treatment response.

The group previously developed an immunocompetent genetically engineered mouse melanoma model and an adoptive transfer protocol that uses $\mathrm{T}$ cells engineered to recognize the melanocyte-specific antigen silver homolog (Silv; Pmel17; Gp100) ${ }^{6-8}$ Similar to patients who are treated with engineered $\mathrm{T}$ cells, tumors in this mouse model initially respond to therapy but often start growing again after two months.

Now, the researchers have used the mouse model to test an adoptive transfer protocol and elucidate the resistance mechanisms that lead to tumor relapse. They found that acquired resistance to $\mathrm{T}$ cell therapies stems from dedifferentiation and downregulated expression of multiple melanocytic antigens. The class of melanocytic antigens includes GP100, which is normally upregulated when cells differentiate into melanocytes (see Figure 1, "Resistance to adoptive T cell therapy via dedifferentiation").

A series of transplantation and cell culture studies showed that the dedifferentiation and antigen loss were reversible and were associated with T cell-driven inflammation. The researchers identified tumor necrosis factor- $\alpha$ (TNF- $\alpha$ ) as the key proinflammatory cytokine responsible for dedifferentiation and antigen loss.

Indeed, $\mathrm{T}$ cells engineered to recognize GP100 or another melanocytic antigen called melan-A (MLANA; MART1) showed less reactivity against
"The key challenge is to further improve treatment protocols by combining adoptive cell transfer with complementary strategies such as checkpoint blockade and inhibitors of oncogenic signaling pathways."

-Thomas Tüting, University Hospital Bonn human melanoma cells exposed to TNF- $\alpha$ than against unexposed melanoma cells. In contrast, two $\mathrm{T}$ cell lines engineered to recognize two different nonmelanocytic antigens showed unchanged and increased reactivity against TNF- $\alpha$ exposed human melanoma cells, respectively.

Results were published in Nature.

"We found that tumor relapse after initially successful T cell immunotherapy involves a reversible adaptive process of dedifferentiation driven by proinflammatory mediators such as TNF- $\alpha$ secreted by tumor-infiltrating immune cells in the tumor microenvironment," said corresponding author Tüting.

"These results are very interesting for adoptive cell therapies, as they could explain why we often see partial responses as opposed to complete responses in the patient's lesions," said Laszlo Radvanyi, a professor in the Department of Melanoma Medical Oncology at The University of Texas MD Anderson Cancer Center. "T cells produce proinflammatory cytokines during the antitumor immune response, and perhaps the TNF- $\alpha$ they produce causes loss of melanosomal antigens that these cells target." 
I. Model of reversible dedifferentiation

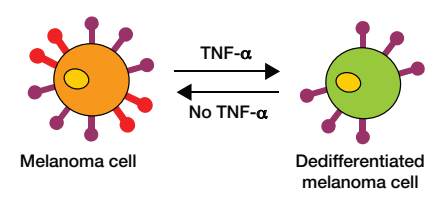

II. Model of acquired resistance to T cell therapy

a
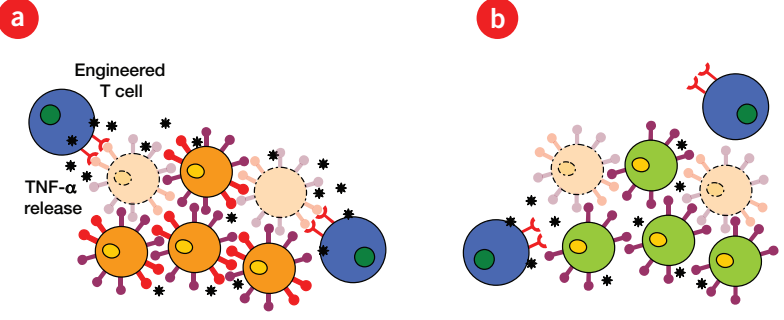

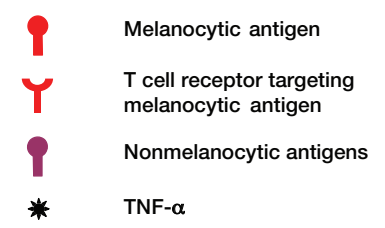

(C)

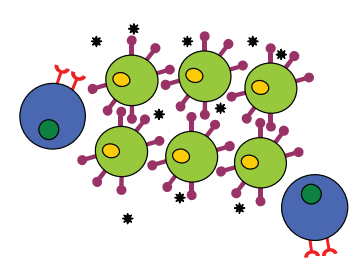

Figure 1. Resistance to adoptive T cell therapy via dedifferentiation. As reported by Landsberg et al., melanoma cells can undergo inflammation-induced dedifferentiation. The process is a potential acquired resistance mechanism against adoptive cell transfer therapies.

(I) Melanoma cells exposed to the proinflammatory cytokine tumor necrosis factor- $\alpha$ (TNF- $\alpha$ ) undergo reversible dedifferentiation. Cells with the dedifferentiated phenotype show downregulated expression of melanocytic antigens.

(II) Adoptive cell transfer therapies for melanoma can involve the infusion of T cells engineered to express antigen receptors that recognize melanocytic antigens. The dedifferentiation mechanism could contribute to resistance against such therapies.

Infused T cells recognize antigens expressed by the melanoma cells and trigger an immune response (II[a]). However, TNF- $\alpha$ released by these T cells during the immune response also can trigger the dedifferentiation process (II[b]). The infused T cells are unable to recognize dedifferentiated melanoma cells. Over time, these dedifferentiated cells regrow and become the dominant cellular phenotype in the tumor (II[c]).

"The most interesting finding of the work was not the immune selection of antigen-specific variants but rather the fact that TNF- $\alpha$, presumably generated from antigen-specific infiltrating effector $\mathrm{T}$ cells, was capable of generating passive resistance," added Jeffrey Weber, a senior member and director of the Donald A. Adam Comprehensive Melanoma Research Center of Excellence at the H. Lee Moffitt Cancer Center \& Research Institute.

According to Antoni Ribas, the findings might explain why adoptive transfer of tumor-infiltrating lymphocytes tends to elicit a more durable tumor response than adoptive transfer of engineered $\mathrm{T}$ cells. The reason is that tumor-infiltrating lymphocytes used in adoptive therapies target a broad range of tumor antigen epitopes and not just a single antigen epitope.

Ribas is a professor in the Department of Medicine at the University of California, Los Angeles David Geffen School of Medicine and director of the tumor immunology program at the university's Jonsson Comprehensive Cancer Center.

\section{From mechanism to therapies}

The key question is whether the mechanism observed in the mouse model applies to melanoma patients who have received adoptive cell transfer.
"I think the data are intriguing, but I would want to hear about what confirmation could be obtained from ongoing adoptive cell transfer trials in melanoma patients," Weber told SciBX.

"If this mechanism applies in humans, and if TNF- $\alpha$ is a key cytokine responsible for reversibly downregulating expression of melanocytic antigens, it does open an opportunity to combine anti-TNF strategies with adoptive cell therapy," added Radvanyi. "What the researchers should do now is show in their model that blocking TNF signaling with an anti-TNF antibody or TNF-targeted shRNA will reverse the dedifferentiation phenomenon."

Ribas cautioned that the systemic immunosuppressive effects of anti-TNF therapies could end up working against the adoptive cell therapy. He thinks a more directed strategy could be to downregulate or prevent TNF- $\alpha$ expression in the transferred T cells themselves.

Ribas said the data in the paper might only apply to melanocytic antigens and not to other classes of melanoma-associated antigens. Thus, he thinks using mixed populations of engineered $\mathrm{T}$ cells that target different classes of antigens could help delay or prevent acquired resistance.

Tüting noted that combining adoptive cell therapies with other immunotherapies and other classes of targeted therapies for melanoma also could improve treatment responses.

"The key challenge is to further improve treatment protocols by combining adoptive cell transfer with complementary strategies such as checkpoint blockade and inhibitors of oncogenic signaling pathways," Tüting told SciBX. "The development of adequate timing and dosing schedules requires a better understanding of the different resistance mechanisms and the establishment of biomarkers and monitoring procedures."

Tüting said the group is working to develop more clinically relevant models that will be useful for testing potential combinations with adoptive cell therapies.

The findings reported in Nature are unpatented.

Lou, K.-J. SciBX 5(42); doi:10.1038/scibx.2012.1100

Published online Oct. 25, 2012

\section{REFERENCES}

1. Landsberg, J. et al. Nature; published online Oct. 10, 2012; doi:10.1038/nature11538

Contact: Thomas Tüting, University of Bonn, Bonn, Germany e-mail: thomas.tueting@ukb.uni-bonn.de

2. Atkins, M.B. et al. J. Clin. Oncol. 17, 2105-2116 (1999)

3. Hodi, F.S. et al. N. Engl. J. Med. 363, 711-723 (2010)

4. Rosenberg, S.A. et al. Clin. Cancer Res. 17, 4550-4557 (2011)

5. Rosenberg, S.A. Nat. Rev. Clin. Oncol. 8, 577-585 (2011)

6. Tormo, D. et al. Cancer Res. 66, 5427-5435 (2006) 


\section{ANALYSIS}

7. Landsberg, J. et al. Pigment Cell Melanoma Res. 23, 649-660 (2010)

8. Kohlmeyer, J. et al. Cancer Res. 69, 6265-6274 (2009)

COMPANIES AND INSTITUTIONS MENTIONED

Amgen Inc. (NASDAQ:AMGN), Thousand Oaks, Calif. Bristol-Myers Squibb Co. (NYSE:BMY), New York, N.Y.

GlaxoSmithKline plc (LSE:GSK; NYSE:GSK), London, U.K.

H. Lee Moffitt Cancer Center \& Research Institute, Tampa, Fla.
Johannes Gutenberg University Mainz, Mainz, Germany National Cancer Institute, Bethesda, Md.

Novartis AG (NYSE:NVS; SIX:NOVN), Basel, Switzerland

University Hospital Bonn, Bonn, Germany

University of Bonn, Bonn, Germany

University of California, Los Angeles David Geffen School of Medicine, Los Angeles, Calif.

The University of Texas MD Anderson Cancer Center, Houston, Texas 